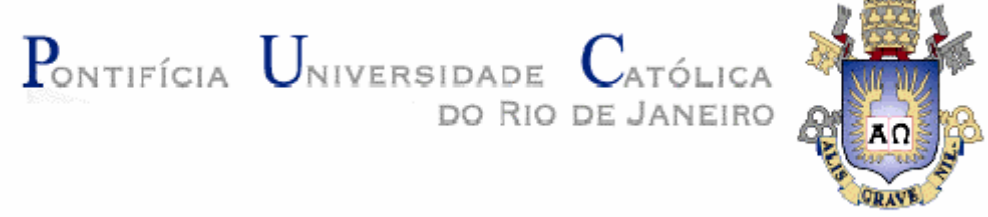

Lina Kátia Mesquita de Oliveira

\title{
TRÊS INVESTIGAÇÕES SOBRE ESCALAS DE PROFICIÊNCIA E SUAS INTERPRETAÇÕES
}

Tese de Doutorado

Tese apresentada ao Programa de PósGraduação em Educação do Departamento de Educação da PUC-Rio como parte dos requisitos parciais para obtenção do título de Doutor em Educação.

Orientador: Prof. Francisco Creso J. F. J únior Co-Orientador: Prof. Tufi Machado Soares 


\title{
Lina Kátia Mesquita de Oliveira
}

\section{TRÊS INVESTIGAÇÕES SOBRE ESCALAS DE PROFICIÊNCIA E SUAS INTERPRETAÇÕES}

Tese apresentada como requisito parcial para obtenção do título de Doutor pelo Programa de Pós-Graduação em Educação do Departamento de Educação do Centro de Teologia e Ciências Humanas da PUC-Rio. Aprovada pela Comissão Examinadora abaixo assinada.

\author{
Profa. Francisco Creso J .F. J únior \\ Orientador \\ Departamento de Educação - PUC-Rio \\ Prof. Tufi Machado Soares \\ Co-orientador \\ UFJ $F$ \\ Profa. Alícia Maria Catalano de Bonamino \\ Departamento de Educação - PUC-Rio \\ Profa. Maria Apparecida C. Mamede Neves \\ Departamento de Educação - PUC-Rio \\ Prof. Manuel Palácios da Cunha e Melo \\ UFJ $F$ \\ Prof. Ruben Klein \\ Fundação Cesgranrio \\ Prof. Amaury Patrick Gremaud \\ USP \\ Prof. Paulo Fernando C. de Andrade \\ Coordenador Setorial do Centro de \\ Teologia e Ciências Humanas
}

Rio de J aneiro, 29 de agosto 2008. 
Todos os direitos reservados. É proibida a reprodução total ou parcial do trabalho sem autorização da universidade, da autora e do orientador.

\section{Lina Kátia Mesquita de Oliveira}

Graduou-se em Matemática pela Faculdade de Filosofia, Ciências e Letras de Belo Horizonte (FAFIBH) - 1976. É Especialista em Educação Matemática pela Universidade Federal de Juiz de Fora (UFJF) - 1999. É Mestre em Educação pelo Programa de Pós-Graduação da UFJ F, tendo defendido a Dissertação em junho de 2002. Atualmente é Coordenadora da Unidade de Avaliação do Centro de Políticas Públicas e Avaliação da Educação da UFJ F. Atua em proj etos na área de Avaliação de Sistemas Educacionais e de Gestão da Educação.

Ficha Catalográfica

Oliveira, Lina Kátia Mesquita de

Três investigações sobre escalas de proficiência e suas interpretações / Lina Kátia Mesquita de Oliveira ; orientador: Francisco Creso J. F. Júnior ; co-orientador: Tufi Machado Soares. -2008.

216 f. : il. ; $30 \mathrm{~cm}$

Tese (Doutorado em Educação)-Pontifícia Universidade Católica do Rio de Janeiro, Rio de Janeiro, 2008.

Inclui bibliografia

1. Educação - Teses. 2. Avaliação educacional. 3. Escalas de proficiência. 4. Análise de conglomerado. 5. Níveis de proficiência escolar. 6. Interpretação pedagógica de itens. I. Franco, Creso. II. Soares, Tufi Machado. III. Pontifícia Universidade Católica do Rio de Janeiro. Departamento de Educação. IV. Título.

CDD: 370 


\section{Agradecimentos}

Nesse momento, meu objetivo é externar minha gratidão, um dos mais nobres sentimentos expressos pela humanidade, e fazer com que todos aqueles que contribuíram para que eu chegasse até aqui possam permanecer na memória.

Ao meu orientador, Professor Creso Franco, de quem tive a honra de merecer a confiança e compartilhar do seu compromisso com a qualidade e a propriedade da pesquisa educacional. A ele devo a pergunta adequada, o tema desafiador, a inquietação para buscar 0 caminho mais rico e proveitoso. Sério e competente, deixará sua marca no movimento das universidades brasileiras pela excelência na área da avaliação da educação.

Ao Professor Tufi Machado Soares, meu co-orientador e construtor dos modelos estatísticos adotados na pesquisa. Conhecedor profundo das análises de dados quantitativos, dispôs-se a buscar comigo o caminho mais adequado à pesquisa proposta. Seu trabalho, amplamente conhecido, certamente acrescentou dose significativa de valor a esta tese.

Ao Manuel Palacios da Cunha e Mello, responsável por minha formação acadêmica, profissional e, especialmente, por minha capacidade de sonhar e acreditar, generosamente, que o mundo pode ser melhor. Tenho muito orgulho de participar com ele da construção de um centro de estudos e pesquisas que se constituiu como o projeto mais inovador 
na área da avaliação educacional no Brasil, justamente por ser um espaço de formação, de parceria e de excelência científica e tecnológica.

Aos professores participantes das bancas de qualificação, Alícia Bonamino, Fátima Alves, Aparecida Mamede, Amaury Patrick Gremaud, agradeço a preciosa colaboração, as idéias criativas e a disponibilidade.

Tenho muita honra de poder contar, em minha banca, com o Professor Rubem Klein, referência obrigatória e autoridade reconhecida pelo seu trabalho precursor na construção e desenvolvimento de programas de avaliação educacional em larga escala.

Não poderia deixar de registrar, nos meus agradecimentos, a contribuição institucional dada

- pelo Departamento de Educação da PUC-Rio pela qualidade dos cursos e excelência na formação acadêmica e profissional que propicia a seus alunos;

- pela Diretoria de Avaliação da Educação Básica do Instituto Nacional de Pesquisa Anísio Teixeira, do Ministério da Educação, pela disponibilização da base da dados do SAEB;

- pelo CAEd / UFJ F, meu local de trabalho, referência nacional na área da avaliação educacional, onde teoria e prática se associam em busca do melhor debate e da mais cuidadosa interpretação dos temas relevantes da educação pública.

À equipe de pesquisa e análise de dados do CAEd, agradeço pela acolhida carinhosa e sempre disponível. Agradeço especialmente ao Neimar, ao Wellington e ao Luís Fajardo, que me acompanharam mais de perto, me apoiando nesta caminhada. Muitas vezes transformaram minhas dificuldades no campo da estatística em idéias claras, objetivas e promissoras. Ao Ailton e ao Helson, agradeço pelo desenvolvimento dos sistemas operacionais utilizados. 
A Magda, Ederaldo, J anine, Verônica, Isabela, Regina, Lulude, Claytom e Márcio pelo apoio e aj uda constante em todos os momentos.

À minha amiga Wanda Alves, pela disponibilidade para ler, observar e opinar na área da educação matemática.

À Luiza que, com muito carinho e competência nas artes gráficas, editorou o trabalho, não se poupando mesmo nos fins de semana.

À Shirley Torres e à J osiane Toledo pela disponibilidade, apoio e revisão dos textos.

Aos meus queridos, Tio Raimundo e Tia Zezé, e às queridas amigas Lucy, Marlene, Bia e Letícia. Obrigada pela confiança, palavras de incentivo e pela torcida.

À Eleuza, minha irmã emprestada, companheira e amiga, que me ofereceu o olhar confiante, a palavra de incentivo e a mais rica oportunidade da vida: poder contar com a pessoa certa na hora certa, o meu agradecimento pela valiosa colaboração na revisão desse trabalho.

Por fim, de modo muito especial e carinhoso agradeço à minha família pelo companheirismo, solidariedade e força com que me presentearam. Apoio incondicional, paciência e segurança. Obrigada, José Francisco, Fernando e Mayra, vocês realmente são uns amores.

Dedico esse trabalho à minha mãe. Tenho certeza que, onde estiver, ol ha por mim, me abençoa e protege. 


\section{Resumo}

Oliveira, Lina Kátia Mesquita de; Franco Jr., Francisco Creso J unqueira. Três Investigações sobre Escalas de Proficiência e suas Interpretações. Rio de J aneiro, 2008. 216 p. Tese de Doutorado Departamento de Educação, Pontifícia Universidade Católica do Rio de J aneiro.

As avaliações de proficiência em larga escala vêm exercendo um papel relevante no contexto educacional contemporâneo, pois servem, entre outros propósitos, para retratar mais precisamente o nível de desempenho escolar de uma grande quantidade de alunos avaliados, como também para fornecer subsídios mais sólidos ao aperfeiçoamento das políticas educacionais que vêm sendo implementadas. As escalas de proficiência utilizadas nessas avaliações revestem-se de uma grande importância nesse processo, visto que são utilizadas pelos avaliadores e pelos gestores educacionais como um instrumento capaz de aferir e interpretar a proficiência dos alunos nas diversas habilidades que compõem os domínios de conhecimento investigados. 0 objeto de estudo da presente tese são precisamente as escalas de proficiência utilizadas nos sistemas de avaliação em larga escala, que são tratadas em três diferentes abordagens: primeiro, há uma avaliação das diferentes abordagens para se obter uma associação entre os itens de um teste e os níveis ou pontos significativos de proficiência de diversas escalas atualmente empregadas. Depois, utilizando uma escala de proficiência em Matemática para a quarta série do Ensino Fundamental, é desenvolvida uma abordagem alternativa para caracterizar os níveis representativos de uma escala de proficiência escolar. Para isso foram utilizados métodos baseados em análise de conglomerados (cluster analysis) para a escolha do subconjunto de itens que caracteriza cada nível de proficiência. Por fim, é realizada uma interpretação pedagógica dos clusters formados, com base nos conhecimentos do ensino e da aprendizagem das habilidades básicas da educação matemática para a primeira etapa do ensino fundamental. A abordagem utilizada será a análise da natureza das operações mentais que caracterizam cada uma das competências e habilidades que compõem os clusters, tendo como pressupostos básicos as referências ao processo de aprendizagem da matemática.

\section{Palavras-chave:}

Avaliação educacional; escalas de proficiência; análise de conglomerado; níveis de proficiência escolar; interpretação pedagógica de itens. 


\section{Abstract}

Oliveira, Lina Kátia Mesquita de; Franco Jr, Francisco Creso J unqueira. Three Investigations of Proficiency Scales and Their Interpretation. Rio de Janeiro, 2008. 216 p. Tese de Doutorado Departamento de Educação, Pontifícia Universidade Católica do Rio de J aneiro.

Large-scale evaluations have played a relevant role in the contemporary field of education, not only because of their importance in providing a more precise assessment of the proficiency of large numbers of students, but also because of their use as a means of producing more reliable elements that are used in the improvement of educational policies. The proficiency scales adopted in these evaluation programs are a very important part of this process, for they are employed by educational evaluators and decision-makers as an instrument able to gauge and interpret the students' levels of proficiency in the many different abilities that compose the knowledge domains under investigation. The object of this thesis is precisely the proficiency scales used in large scale evaluation systems, which are treated according to three different approaches: First an investigation is made about the relative merit of different methods of association between the items that make up a test and the significant points or levels of proficiency in several scales that have been employed by different evaluation programs. Then, by using a 4th-grade Mathematics proficiency scale, an alternative approach to characterize levels that are representative of a proficiency scale is developed. This is done by the use of a cluster analysis method aimed at choosing a subset of items that characterize each proficiency level. Finally, a pedagogical interpretation of the clusters formed along the proficiency scale is obtained through the consideration of teaching and learning of Mathematics education basic skills, related to the beginning series of the fundamental level. The methodology used in this process is the analysis of the nature of mental operations that characterize each one of the competencies and skills that are typical of each cluster, by adopting references to Mathematics learning processes as basic assumptions.

\section{Key-words:}

Educational evaluation; proficiency scales; cluster analysis; school proficiency levels; pedagogical interpretation of items. 


\section{Sumário}

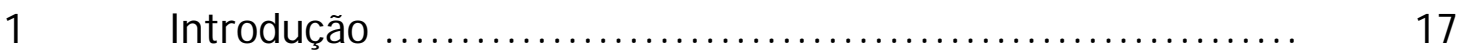

2 Avaliação das diferentes abordagens para a associação entre itens e níveis ou pontos de uma escala de proficiência................................................ 22

2.1 Conceitos básicos: dos itens à Teoria da Resposta ao Item ..... 24

2.1.1 As principais características dos itens utilizados nas avaliações de larga escala ................................ 26

2.1.2 Aspectos básicos da Teoria da Resposta ao Item (TRI) ......... 27

2.1.3 Os parâmetros da TRI .................................... 30

2.2 As Escalas de Proficiência .................................. 34

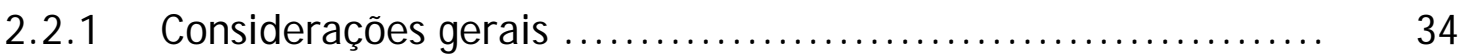

2.2.2 Transformações lineares ................................. 36

2.2.3 Dando significado às Escalas de Proficiência .................. 37

2.2.4 Construção da Escala de Proficiência: critérios de seleção de

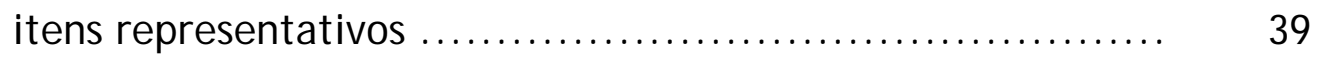

2.3 Principais referências de abordagens para a associação entre itens e níveis ou pontos característicos de uma escala de proficiência ............................................. 40

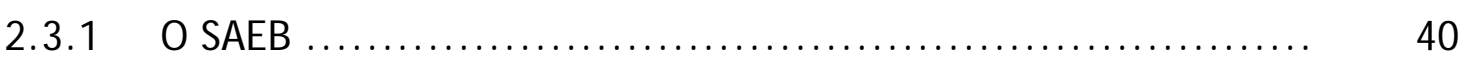

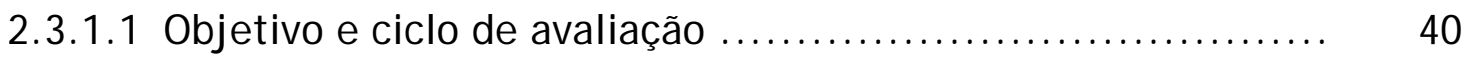

2.3.1.2 A seleção de itens representativos utilizada pelo SAEB a partir de 1999 
2.3.1.3 A seleção de itens representativos utilizada pelo SAEB em 1995 e 1997.

2.3.2 Análise comparativa entre os critérios de seleção utilizados pelo SAEB a partir de 1999 e os utilizados em 1995 / 1997 .... 46

2.4 O NAEP: objetivo e ciclo de avaliação ....................... 49

2.4.1 A ancoragem de itens utilizada pelo NAEP ................... 50

2.4.2 Análise comparativa entre os critérios de seleção utilizados pelo SAEB a partir de 99 e o NAEP ......................... $\quad 59$

2.5 O TIMSS: objetivo e ciclo de avaliação ........................ 60

2.5.1 A seleção de itens representativos utilizada pelo TIMSS ........ 62

2.5.2 Considerações sobre a seleção dos itens segundo os critérios do TIMSS ................................................ 65

2.5.3 Análise comparativa entre os critérios de seleção utilizados pelo SAEB a partir de 99 e 0 TIMSS .......................... 66

2.6 O PISA: objetivo e ciclo de avaliação ......................... 67

2.6.1 A seleção de itens representativos utilizada pelos PISA ........ 68

2.6.2 Análise comparativa entre os critérios de seleção utilizadas pelo SAEB a partir de 1999 e os critérios de interpretação de escala do PISA .......................................... 73

2.7 O Projeto GERES: Objetivos e ondas de aplicação .............. 75

27.1 Metodologia de construção da escala de proficiência adotada

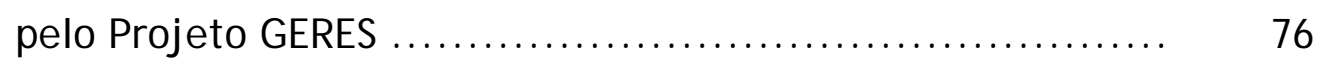

2.7.2 Análise comparativa entre os critérios de seleção utilizados pelo SAEB a partir de 99 e o GERES $\ldots \ldots \ldots \ldots \ldots \ldots \ldots \ldots \ldots . . \ldots$

2.7.2.1 Comparações entre o SAEB 1999-2007 e o GERES no ponto de auge do desenvolvimento de habilidade ..................... 81

2.7.2.2 Comparações entre o SAEB 1999-2007 e o GERES no ponto de consolidação da habilidade

30 uso da análise de conglomerados na seleção de um conjunto de itens característicos dos níveis de proficiência ... $\quad 92$

3.1 Análise de clusters: considerações gerais ...................... 93

3.1.1 Formação dos agrupamentos .............................. 93 
3.2 Construção de clusters para a seleção de itens característicos dos níveis da escala de proficiência

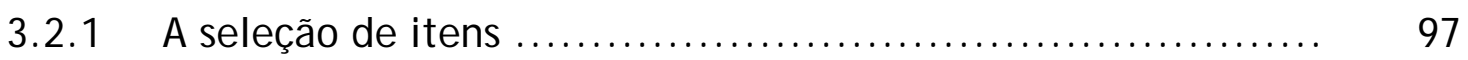

3.2.2 0 agrupamento inicial dos itens selecionados ................. 98

3.2.3 Características gerais do agrupamento de itens após a obtenção da solução com 6 clusters ......................... 109

4 Interpretação pedagógica da Escala de Proficiência a partir dos resultados dos clusters ................................ 118

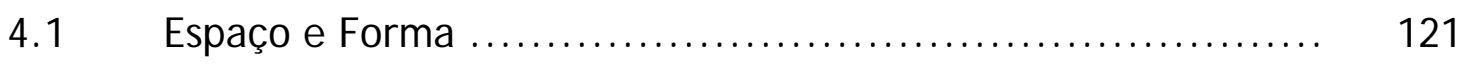

$4.2 \quad$ Medidas e Grandezas ...................................... 124

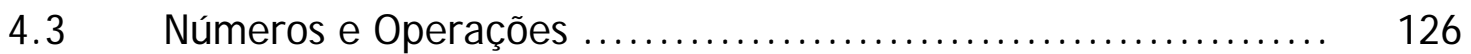

$4.4 \quad$ Tratamento da Informação ............................... 128

4.5 Interpretação Pedagógica dos clusters de proficiência ......... 129

$5 \quad$ Considerações finais ..................................... 139

$6 \quad$ Referências bibliográficas ................................. 146

Anexos .............................................. 148 


\section{Lista de tabelas}

Tabela 1 Número de itens selecionados por nível de proficiência, segundo os critérios do SAEB 1999-2007

Tabela 2 Número de itens selecionados por nível de proficiência, segundo os critérios do SAEB 1995-1997

Tabela 3 Número de itens-âncora por nível de proficiência conforme o primeiro critério do NAEP

Tabela 4 Número de itens-âncora por nível de proficiência conforme o segundo critério do NAEP....

Tabela 5 Pontos selecionados na escala TIMSS

Tabela 6 Níveis selecionados na escala TIMSS

Tabela 7 Correspondência entre os níveis de proficiência do TIMSS e do SAEB nos níveis de seleção definidos pelos critérios do TIMSS

Tabela 8 Número de itens selecionados por nível de proficiência conforme os três critérios do TIMSS considerados separadamente

Tabela 9 Número de itens selecionados por nível de proficiência conforme os três critérios do TIMSS tomados conjuntamente

Tabela 10 Níveis e intervalos de proficiência no PISA ....

Tabela 11 Níveis de proficiência no PISA 
Tabela 12 Número de itens selecionados de acordo com cada um dos três critérios do PISA .................................... 73

Tabela 13 Disparidade entre os níveis de seleção segundo os critérios do SAEB (1999-2007) e do GERES (no auge do desenvolvimento da habilidade)

Tabela 14 Número de itens selecionados por nível de proficiência, segundo os critérios do GERES (no auge do desenvolvimento da habilidade)

Tabela 15 Disparidade entre os níveis de seleção segundo os critérios do SAEB (1999-2007) e do GERES (consolidação da habilidade)

Tabela 16 Número de itens selecionados por nível de proficiência, segundo os critérios do GERES (na consolidação da habilidade)

Tabela 17 Resumo dos 11 clusters originais

Tabela 18 Os 6 clusters formados

Tabela 19 Distribuição dos alunos pelos níveis de habilidades 


\section{Lista de quadros}

Quadro 1 Posicionamento dos itens na escala/ critérios de seleção do SAEB - 1999 A 2007

Quadro 2 Posicionamento dos itens na escala/ critérios de seleção do SAEB - 1995 a 1997

Quadro 3 Critérios/Posicionamento dos itens na Escala de Proficiência - NAEP

Quadro 4 Posicionamento dos itens na escala de proficiência/ critérios de seleção - TIMSS

Quadro 5 Avaliação das três fases de desenvolvimento das habilidades

Quadro 6 Distribuição dos alunos da 4a série EF - Prova Brasil 2005 ..

Quadro 7 Níveis de habilidade e proficiências características

Quadro 8 Variação observada entre os diferentes programas associada à quantidade de itens selecionados por cada critério

Quadro 9 Amplitude dos níveis de seleção constituídos de forma contínua, ou descontínua

Quadro 10 Adoção de relaxamentos dos critérios de seleção

Quadro 11 Critério para a Seleção dos Itens Característicos dos Níveis de Proficiência 


\section{Lista de figuras, gráficos e diagramas}

Figura 1 :Cls de quatro itens de diferentes níveis de dificuldade, segundo o modelo de um parâmetro da TRI

Figura 2 CCls de quatro itens com variações de dificuldade e de discriminação, segundo o modelo de dois parâmetros da TRI

Figura 3 CCls de quatro itens com variações de dificuldade e de discriminação, levando-se em conta também o acerto casual, segundo o modelo de três parâmetros da TRI .....

Figura 4 A curva normal de proficiência 35

Figura 5 Representação gráfica de Beaton e Allen

Figura 6

Item 13099

Figura 7 Item 25099

Figura 8 Fases de desenvolvimento das habilidades

Figura 9 Exemplo hipotético de Curva Característica do Item ......

Figura 10 Curva Característica do Item e Curva de Informação do Item (CII) para um item testado no GERES

Figura 11 Dendograma

Figura 12 Aplicação de k-means

Figura 13 Exemplo de uma Curva Característica do Item

Gráfico 1 Número de itens selecionados segundo os diferentes critérios considerados 


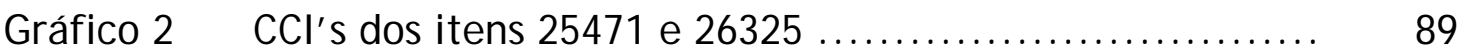

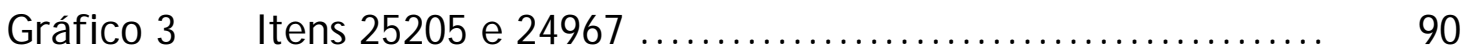

Gráfico 4 Itens com seus respectivos pontos notáveis próximos, o que produz uma distância euclidiana menor e torna-os similares .. 101

Gráfico 5 Itens com seus respectivos pontos notáveis mais distantes entre si e, conseqüentemente, menos similares .............. 101

Gráfico 6 Distribuição do número de itens ao longo dos 11 clusters formados ........................................... 105

Gráfico 7 Distribuição dos itens ao longo dos 11 clusters formados . 105

Gráfico 8 Distribuição dos 11 clusters formados ................. 106

Gráfico 9 Representação bidimensional das centróides dos 11 clusters formados e seus respectivos itens .............. 108

Gráfico 10 Distribuição do número de itens ao longo dos 6 clusters formados

Gráfico 11 Distribuição dos itens ao longo dos 6 clusters formados..

Gráfico 12 Distribuição dos 6 clusters formados

Gráfico 13 Representação bidimensional das centróides dos 6 clusters formados e seus respectivos itens

Diagrama 1 Escala de Proficiência em Matemática - Primeira etapa do Ensino Fundamental 